\title{
The "Ockham's razor" approach to diseases and patients: viruses from the picornaviridae family and related diseases
}

\author{
Jevtović $\mathrm{Dj}^{*}$ \\ HIV/AIDS Department, Institute for Infectious \& Tropical Diseases, Clinical Centre of Serbia, Belgrade University School of Medicine, Serbia
}

Entroviruses, the member of picornavirida family, have become invisible relying on a totally different evolution strategy then the rhinoviruses that we can unmistakeably identify. The most important members of the picornaviridae family affecting the humans are rhinoviruses with over one hundred serotypes, and much less numerous entroviruses.

During their evolution along with its natural host, thehumans, rhinoviruses have already managed to cause some damage to the host and still to replicate, change rapidly when necessary, even though with certain causalities, spread easily, finding new susceptible hosts. Accordingly, they have already reached the viral antechamber.

What has happened with their relatives, the enteroviruses? Since enteroviruses are causing a wide spectrum of clinical conditions, from common cold, herpangina and rush, mostly benign paediatric diseases, to much more serious myopericarditis, either in paediatric population, or among adults and occasionally elderly people, it appears that enteroviruses have forgotten that diseases are accidents, the episodes which may kill both the microorganism and the host; both undesirable. All of the diseases mentioned above could be caused by many other viral and/or bacterial pathogens, related to patient's age and immune competence. The history of infections can easily recall that President Roosevelt suffered complicated poliomyelitis in his advanced age. Nobody knows how many similar cases have happened ever since among elderly patients caused by other members of the enteroviruses family, and how many of them were misdiagnosedas cerebrovascular events, multiple sclerosis (MS), which areindeed much more prevalent among certain patients populationscontrary to entroviral infections that are seldom life threatening. Taking a good history from a patent is the clue toeasily recognizethese enteroviral-related neurologicand other conditions. They have a biphasic course, with a febrile introduction, as opposed to acute cerebrovascular events. Unfortunately, MS could sometimes take a biphasic course and accordingly be misdiagnosed. It was shown not to be uncommon that some patients who sufferedMSlike diseases recovered spontaneously, instead of progressing to typical chronic undulating life threatening neurologic condition. What is going on nowadays with poliovirus relatives? A few years ago, an unusual outbreak of polio-like disease was described among Californian children attending a kindergarten, caused by Enterovirus 68. Similar episodesare most probably everyday routine occurrence in many children clinics and hospitals, but they come and go unrecognized. The same applies to many immunocompetent and immunocompromised children and

Copyright: ${ }^{\circ} 2018 \mathrm{Dj}$ J. This is an open-access article distributed under the terms of the Creative Commons Attribution License, which permits unrestricted use, distribution, and reproduction in any medium, provided the original author and source are credited. adults who suffer strange neurologic conditions, including aseptic meningitis and/or encephalitis, or even militias, also etiologically undiagnosed, since these usually undertook a benign self limited course. Neurologist and infectious diseases specialists have forgotten that the easiest way to come to the conclusion and diagnosis is to test patients stool in order to detect enteroviral RNA, using PCR technique, which is feasible, even though not standardised and commercially available always and everywhere. In many instances this is much more accurate then more non-specific an expensive CT, MRI, and/or PET scans. A good history, physical examination and few easy to perform laboratory analyses, along with ordinary neuroimmaging techniques are feasible and mostly available to rule out serious diseases over those with more benign and often self-limited course. This kind of approach to patients and diseases is easy and is cost effective in terms of both money and health resources.

Currently, the most interesting entroviral related disease is a newborn septicaemia-like disease, which is always life threatening, and difficult to distinguish from more common neonatal conditions, which may be caused by several bacterial pathogens, including Escherichia coli, Lysteria monocytogenes, and group B streptococcus, all of which are inhabitants of the birth channel, along with herpes simplex virus type 2. All these microbes, orat least most of them, are causing a lifethreatening Multiple Organ Dysfunction Syndrome (MODS) in an initially healthy newborn, since all of them have an incubation period of quite a number of days, which is needed for certain pathogens to replicate on the respiratory or GIT mucosa, to reach RES, and eventfully cause the end organs disease.

\section{References}

1. Melnick JL (1983) Portraits of viruses: the picornaviruses. Intervirology 20: 61-100. [Crossref]

2. AtmarRL, Piedra PA, Patel SM, Greenberg SB, Couch RB, et al. (2011) Picornavirus the Most Common Respiratory Virus Infection among Patients of All Ages Hospitalized with Acute Respiratory Illness. J ClinMicrobiol 50: 506-508.

3. Racaniello VR (2006) Picornaviridae: the viruses and their replication. Knipe DM, Howley PM, Griffin DE. Fields Virology (5th Edn) Lippincott Williams \& Wilkins 1: 795-838

4. Melnick JL (1992) Properties and classification of hepatitis A virus. Vaccine 10 Suppl 1: S24-26. [Crossref]

5. Papadopoulos NG, Sanderson G, Hunter J (1999) Rhinoviruses replicate effectively at lower airway temperatures. J Med Virol 58: 100-104.

*Correspondence to: Jevtović Dj, HIV/AIDS Department, Institute for Infectious \& Tropical Diseases, Clinical Centre of Serbia, Belgrade University School of Medicine, Bulevar Oslobodjenja 16, 11000 Belgrade, Serbia, E-mail: djordjejevtovic@hotmail.com

Received: September 12, 2018; Accepted: September 25, 2018; Published: September 28, 2018 